\title{
Production of heavy charged particles in proton-proton ultraperipheral collisions at the Large Hadron Collider: survival factor
}

\author{
S.I. Godunov, ${ }^{a}$ V.A. Novikov, ${ }^{a}$ A.N. Rozanov, ${ }^{b, c}$ M.I. Vysotsky ${ }^{a}$ and \\ E.V. Zhemchugov ${ }^{a}$
}

${ }^{a}$ I.E. Tamm Department of Theoretical Physics, Lebedev Physical Institute, 53 Leninskiy Prospekt, Moscow, 119991, Russia

${ }^{b}$ A.I. Alikhanov Institute for Theoretical and Experimental Physics (ITEP) of National Research Centre 'Kurchatov Institute',

25 Bolshaya Cheremushkinskaya, Moscow, 117218, Russia

${ }^{c}$ Centre de Physique des Particules de Marseille (CPPM), Aix-Marseille Universite, CNRS/IN2P3, 163 avenue de Luminy, case 902, Marseille, 13288, France

E-mail: sigodunov@lebedev.ru, novikovitep@gmail.com, rozanov@cppm.in2p3.fr, vysotskymi@lebedev.ru, evgenii.zhemchugov@cern.ch

ABSTRACT: Ultraperipheral collisions of high energy protons are a source of approximately real photons colliding with each other. Photon fusion can result in production of yet unknown charged particles in very clean events. The cleanliness of such an event is due to the requirement that the protons survive during the collision. Finite sizes of the protons reduce the probability of such outcome compared to point-like particles. We calculate the survival factors and cross sections for the production of heavy charged particles at the Large Hadron Collider.

Keywords: QCD Phenomenology

ARXIV EPRINT: 2106.14842 


\section{Contents}

1 Introduction 1

2 EPA and the survival factor $\quad 2$

$\begin{array}{lll}3 & \text { Production of heavy charged particles } & 7\end{array}$

4 Comparison to the experimental data 11

5 Conclusion 12

\section{Introduction}

Protons accelerated at the LHC are the sources of strong electromagnetic fields carried by almost real equivalent photons. The idea to consider the LHC as a photon-photon collider was analyzed in detail long ago [1-15]. If new charged particles exist, they should be produced in ultraperipheral collisions (UPC) of protons. The corresponding cross section is model independent and is determined by masses and electric charges of these particles.

Finite sizes of protons modify the equivalent photons approximation developed for point-like colliding particles. First, the equivalent photons flux is reduced by the elastic form factor. Second, the protons should miss each other to avoid inelastic interaction and loss of coherence. The corresponding reduction of the total cross section of the UPC production of definite final state depends on a single variable $r_{p} \sqrt{s} / \gamma$, where $\sqrt{s}$ is the invariant mass of the created system, $r_{p}$ is the proton radius, and $\gamma$ is the Lorentz factor of each proton [16]. ${ }^{1}$ This is the reason why the survival factor, being at the level of few percents in the case of the production of a muon pair, considerably diminishes the cross section for the production of heavy particles.

To take into account the reduction of the photon flux, we use the Weizsäcker-Williams equivalent photons approximation (EPA) in the impact parameter space. Our approach is close to that of ref. [16], where one can find references to the earlier literature devoted to this problem. However, we are taking into account elastic form factors explicitely, while in ref. [16] the nucleus interior is completely excluded: integrations over impact parameters $b_{i}$ go from $r_{p}$ to infinity.

In the framework of electroweak theory, diagrams in which one or both intermediate photons are replaced by $Z$ bosons also contribute to the particles production. At the amplitude level, replacement of a photon by $Z$ results in the suppression factor of the order

\footnotetext{
${ }^{1}$ Dimensionless reduction of the total cross section depends on two dimensionful quantities: $r_{p}$ and $\sqrt{s}$. Photon energy $\omega$ in EPA is always divided by $\gamma$. The corresponding scaling behaviour follows. When additional scales are introduced (e.g. cuts), this simple behaviour is no longer correct.
} 
of $\left(\Lambda_{\mathrm{QCD}} / m_{Z}\right)^{2} \sim 10^{-6}$ as soon as we demand that the proton remains intact after emitting the $Z$ boson. Consequently, contributions of intermediate $Z$ into the UPC processes considered in the present paper are completely negligible.

In section 2 explicit formulae which take into account finite sizes of the colliding particles are derived. They are applied in section 3 to the production of heavy charged particles and in section 4 to the production of $\mu^{+} \mu^{-}$pairs in proton-proton collisions. We conclude in section 5 .

\section{EPA and the survival factor}

The spectrum of equivalent photons of a proton with the Lorentz factor $\gamma$ is [17-19]

$$
n(b, \omega)=\frac{\alpha}{\pi^{2} \omega}\left[\int_{0}^{\infty} \frac{F\left(q_{\perp}^{2}+\omega^{2} / \gamma^{2}\right)}{q_{\perp}^{2}+\omega^{2} / \gamma^{2}} J_{1}\left(b q_{\perp}\right) q_{\perp}^{2} \mathrm{~d} q_{\perp}\right]^{2},
$$

where $n(b, \omega) \mathrm{d}^{2} b \mathrm{~d} \omega$ is the number of photons with the energy $\omega$ moving through a point of space with the impact parameter $\vec{b}, \alpha$ is the fine structure constant, $q_{\perp}$ is the transverse photon momentum, $q_{\perp}^{2}+\omega^{2} / \gamma^{2} \equiv Q^{2}$ is the photon virtuality, $F\left(Q^{2}\right)$ is the Dirac form factor of the proton, $J_{1}(z)$ is the Bessel function of the first kind.

With the help of the relation

$$
\int_{0}^{\infty} J_{1}(a x) J_{1}(b x) x \mathrm{~d} x=\frac{1}{a} \delta(a-b),
$$

integration of $n(b, \omega)$ over the whole impact parameter space $\mathrm{d}^{2} b$ results in the following well known formula for the spectrum of equivalent photons:

$$
n(\omega)=\int n(b, \omega) \mathrm{d}^{2} b=\frac{2 \alpha}{\pi \omega} \int_{0}^{\infty}\left[\frac{F\left(q_{\perp}^{2}+\omega^{2} / \gamma^{2}\right)}{q_{\perp}^{2}+\omega^{2} / \gamma^{2}}\right]^{2} q_{\perp}^{3} \mathrm{~d} q_{\perp} .
$$

The proton Dirac form factor is $[20,21]$

$$
F\left(Q^{2}\right)=\frac{G_{E}\left(Q^{2}\right)+\tau G_{M}\left(Q^{2}\right)}{1+\tau}
$$

where $G_{E}\left(Q^{2}\right)$ and $G_{M}\left(Q^{2}\right)$ are known as the Sachs electric and magnetic form factors, $\tau=$ $Q^{2} / 4 m_{p}^{2}, m_{p}$ is the proton mass. In ultraperipheral collisions $Q^{2} \lesssim \Lambda_{\mathrm{QCD}}^{2} \sim 0.04 \mathrm{GeV}^{2}$ [19], so $\tau \lesssim 0.01 \ll 1$, and the magnetic contribution can usually be neglected. However, our calculations have shown that the magnetic form factor contribution to the photon-photon luminosity is at the same order of magnitude as the survival factor contribution, so we keep it in the following.

One common description for the electric and magnetic form factors is the dipole approximation

$$
G_{E}\left(Q^{2}\right)=\frac{1}{\left(1+Q^{2} / \Lambda^{2}\right)^{2}}, \quad G_{M}\left(Q^{2}\right)=\frac{\mu_{p}}{\left(1+Q^{2} / \Lambda^{2}\right)^{2}}
$$


where $\mu_{p}=2.7928473508(85)$ is the proton magnetic moment [22], and the standard value of $\Lambda^{2}$ is $\Lambda_{\text {std }}^{2}=0.71 \mathrm{GeV}^{2}$. Relatively recent experimental data disfavor the dipole approximation [23]. However, according to figure 10(a) of ref. [23], the discrepancy between the fit to the experimental data and the standard dipole approximation is at the level of a few percent for $Q^{2} \lesssim 0.04 \mathrm{GeV}^{2}$, with the fit being below the approximation. The slope of $G_{E}\left(Q^{2}\right)$ at $Q^{2}=0$ is related to the charge radius of proton:

$$
r_{p}^{2}=-6 \frac{\mathrm{d} G_{E}\left(Q^{2}\right)}{\mathrm{d} Q^{2}}
$$

With the CODATA value of $r_{p}=0.8751(61) \mathrm{fm}[22]$ (which is in agreement with the value obtained in ref. [23], see eq. (52)), we get another estimation of $\Lambda^{2}$ :

$$
\Lambda_{\text {CODATA }}^{2}=12 / r_{p}^{2}=0.61 \mathrm{GeV}^{2} .
$$

We use this value in the following calculations. ${ }^{2}$

Application of the dipole approximation to eq. (2.1) results in

$$
\begin{aligned}
& n(b, \omega)=\frac{\alpha}{\pi^{2} \omega}\left[\frac{\omega}{\gamma} K_{1}\left(\frac{b \omega}{\gamma}\right)-\left(1+\frac{\left(\mu_{p}-1\right) \frac{\Lambda^{4}}{16 m_{p}^{4}}}{\left(1-\frac{\Lambda^{2}}{4 m_{p}^{2}}\right)^{2}}\right) \sqrt{\Lambda^{2}+\frac{\omega^{2}}{\gamma^{2}}} K_{1}\left(b \sqrt{\Lambda^{2}+\frac{\omega^{2}}{\gamma^{2}}}\right)\right. \\
& +\frac{\left(\mu_{p}-1\right) \frac{\Lambda^{4}}{16 m_{p}^{4}}}{\left(1-\frac{\Lambda^{2}}{4 m_{p}^{2}}\right)^{2}} \sqrt{4 m_{p}^{2}+\frac{\omega^{2}}{\gamma^{2}}} K_{1}\left(b \sqrt{4 m_{p}^{2}+\frac{\omega^{2}}{\gamma^{2}}}\right) \\
& \left.-\frac{1-\frac{\mu_{p} \Lambda^{2}}{4 m_{p}^{2}}}{1-\frac{\Lambda^{2}}{4 m_{p}^{2}}} \cdot \frac{b \Lambda^{2}}{2} K_{0}\left(b \sqrt{\Lambda^{2}+\frac{\omega^{2}}{\gamma^{2}}}\right)\right]^{2},
\end{aligned}
$$

where $K_{0}(z), K_{1}(z)$ are the modified Bessel functions of the second kind (the Macdonald functions). Integration over $\mathrm{d}^{2} b$ yields

$$
\begin{aligned}
n(\omega)=\frac{\alpha}{\pi \omega}\{ & \left(1+4 u-2\left(\mu_{p}-1\right) \frac{u}{v}\right) \ln \left(1+\frac{1}{u}\right) \\
& +\frac{\mu_{p}-1}{(v-1)^{4}}\left[\frac{\mu_{p}-1}{v-1}(1+4 u+3 v)-2\left(1+\frac{u}{v}\right)\right] \ln \frac{u+v}{u+1}-\frac{24 u^{2}+42 u+17}{6(u+1)^{2}} \\
& +\left(\mu_{p}-1\right) \frac{6 u^{2}\left(v^{2}-3 v+3\right)+3 u\left(3 v^{2}-9 v+10\right)+2 v^{2}-7 v+11}{3(u+1)^{2}(v-1)^{3}} \\
& \left.-\left(\mu_{p}-1\right)^{2} \frac{24 u^{2}+6 u(v+7)-v^{2}+8 v+17}{6(u+1)^{2}(v-1)^{4}}\right\}
\end{aligned}
$$

where

$$
u=\left(\frac{\omega}{\Lambda \gamma}\right)^{2}, v=\left(\frac{2 m_{p}}{\Lambda}\right)^{2}
$$

\footnotetext{
${ }^{2}$ Though dipole form factor with $\Lambda_{\mathrm{CODATA}}$ gives much better description of experimental data than with $\Lambda_{\text {std }}$ for $Q^{2} \lesssim 0.04 \mathrm{GeV}^{2}$, let us note that in the region $Q^{2} \gtrsim 0.04 \mathrm{GeV}^{2}$ data points are above dipole approximation with $\Lambda_{\text {CODATA }}$. This may lead to the actual cross section being greater by $\sim 2-3 \%$.
} 


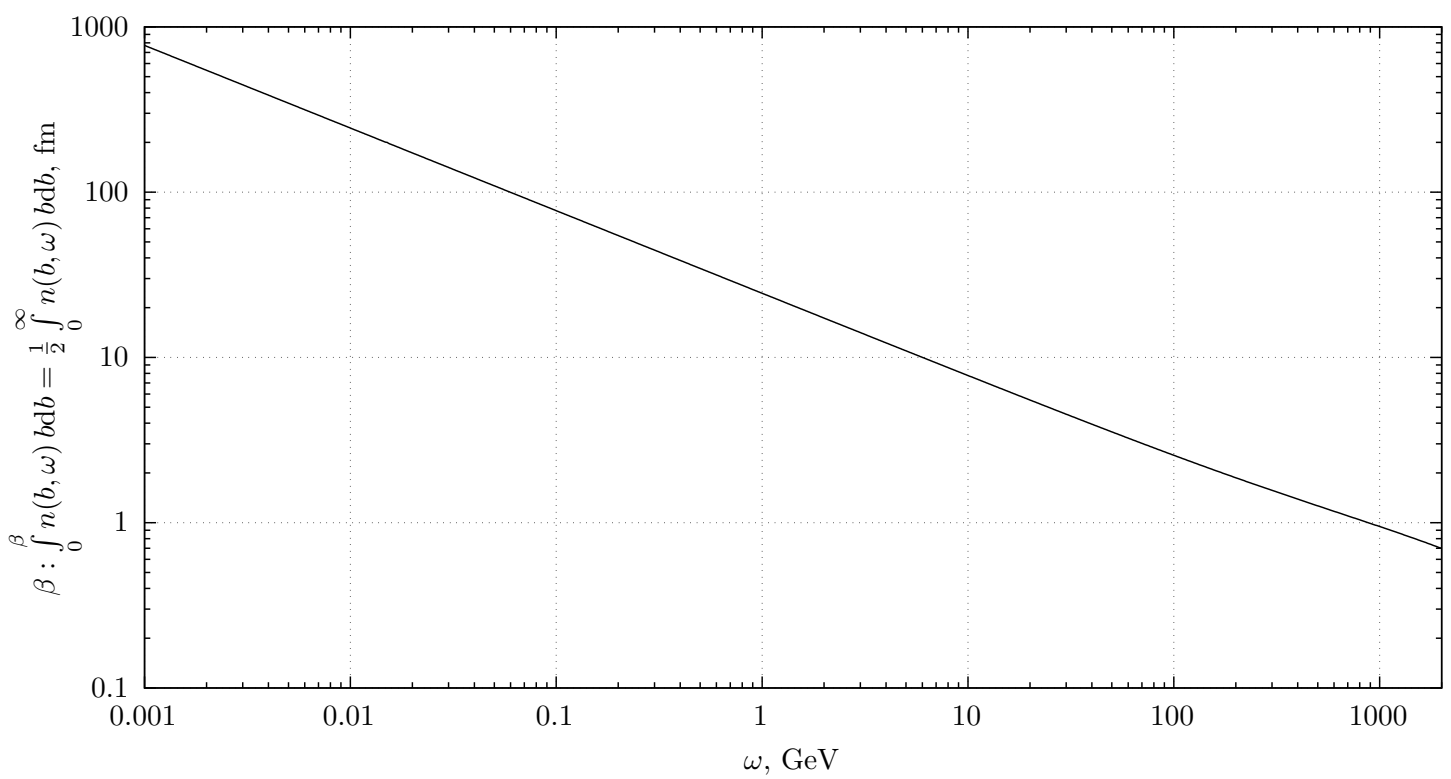

Figure 1. Median of the equivalent photon space distribution with respect to photon energy for a $6.5 \mathrm{TeV}$ proton. For a given photon energy, there is the same amount of photons in the region $r<\beta$ as is in the region $r>\beta$, where $r$ is the distance to the proton center.

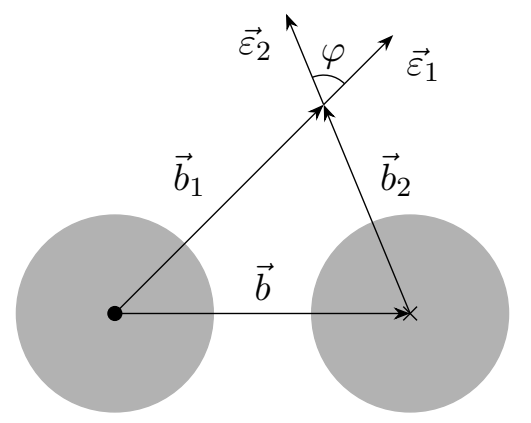

Figure 2. An ultraperipheral collision of two protons moving perpendicular to the figure plane. $\vec{b}_{1}$ and $\vec{b}_{2}$ are impact parameters of a point in space at which photons collide relative to the corresponding protons. $b=\left|\vec{b}_{1}-\vec{b}_{2}\right|$ is the impact parameter of the collision. $\vec{\varepsilon}_{1}$ and $\vec{\varepsilon}_{2}$ are the photon polarization vectors.

To get a grasp on the photon distribution $n(b, \omega)$, see figure 1 , where the median of the equivalent photon space distribution is depicted. The median is defined as such distance $\beta$ that

$$
\int_{b<\beta} \mathrm{d}^{2} b n(b, \omega)=\int_{b>\beta} \mathrm{d}^{2} b n(b, \omega) .
$$

Consider production of a system $X$ in an ultraperipheral collision of two protons. The collision geometry is presented in figure 2 . The cross section is

$$
\sigma(p p \rightarrow p p X)=\int_{0}^{\infty} \mathrm{d} \omega_{1} \int_{0}^{\infty} \mathrm{d} \omega_{2} \int_{b_{1}>\hat{r}} \mathrm{~d}^{2} b_{1} \int_{b_{2}>\hat{r}} \mathrm{~d}^{2} b_{2} \sigma(\gamma \gamma \rightarrow X) n\left(b_{1}, \omega_{1}\right) n\left(b_{2}, \omega_{2}\right) P(b),
$$


where $\sigma(\gamma \gamma \rightarrow X)$ is the $X$ production cross section in photon fusion, and $P(b)$ is the probability for the protons to survive in a collision with the impact parameter $b=\left|\vec{b}_{1}-\vec{b}_{2}\right|$. The integration domain over $\vec{b}_{1}$ and $\vec{b}_{2}$ is limited by the conditions $b_{1}>\hat{r}, b_{2}>\hat{r}$. The value of $\hat{r}$ depends on the nature of the produced system $X$. If $X$ contains strongly interacting particles, as the first approximation one can let $\hat{r}=r_{p}$, the proton charge radius; more accurate treatment of this case requires modification of eq. (2.12) to account for the probability for the particles produced inside a proton to escape. In the following we consider production of particles which do not participate in strong interactions, and we neglect rescattering processes through electromagnetic or weak interactions, so we set $\hat{r}=0$.

Photons in ultraperipheral collisions are polarized in the directions shown in figure 2. Let $\sigma_{\|}(\gamma \gamma \rightarrow X)$ and $\sigma_{\perp}(\gamma \gamma \rightarrow X)$ be the cross sections for the $\gamma \gamma \rightarrow X$ reaction with the photons polarization vectors parallel or perpendicular. These cross sections depend only on the product $\omega_{1} \omega_{2}$, therefore they can be factored out through the change in the integration variables from $\omega_{1}, \omega_{2}$ to $s=4 \omega_{1} \omega_{2}, y=\frac{1}{2} \ln \frac{\omega_{1}}{\omega_{2}}(\sqrt{s}$ is the invariant mass of $X$, $y$ is the rapidity of $X$ ) [24]:

$$
\sigma(p p \rightarrow p p X)=\int_{0}^{\infty} \mathrm{d} s\left[\sigma_{\|}(\gamma \gamma \rightarrow X) \frac{\mathrm{d} L_{\|}}{\mathrm{d} s}+\sigma_{\perp}(\gamma \gamma \rightarrow X) \frac{\mathrm{d} L_{\perp}}{\mathrm{d} s}\right],
$$

where

$$
\begin{aligned}
& \frac{\mathrm{d} L_{\|}}{\mathrm{d} s}=\frac{1}{4} \int_{-\infty}^{\infty} \mathrm{d} y \int_{b_{1}>\hat{r}} \mathrm{~d}^{2} b_{1} \int_{b_{2}>\hat{r}} \mathrm{~d}^{2} b_{2} n\left(b_{1}, \frac{\sqrt{s}}{2} \mathrm{e}^{y}\right) n\left(b_{2}, \frac{\sqrt{s}}{2} \mathrm{e}^{-y}\right) P(b) \cos ^{2} \varphi, \\
& \frac{\mathrm{d} L_{\perp}}{\mathrm{d} s}=\frac{1}{4} \int_{-\infty}^{\infty} \mathrm{d} y \int_{b_{1}>\hat{r}} \mathrm{~d}^{2} b_{1} \int_{b_{2}>\hat{r}} \mathrm{~d}^{2} b_{2} n\left(b_{1}, \frac{\sqrt{s}}{2} \mathrm{e}^{y}\right) n\left(b_{2}, \frac{\sqrt{s}}{2} \mathrm{e}^{-y}\right) P(b) \sin ^{2} \varphi
\end{aligned}
$$

are the photon-photon luminosities for the corresponding relative photon polarizations in UPC of two protons, $\varphi$ is the angle between the vectors $\vec{b}_{1}, \vec{b}_{2}$ as is shown in figure 2 . In the case when the cross section does not depend on the photons polarization, eq. (2.13) simplifies to

$$
\sigma(p p \rightarrow p p X)=\int_{0}^{\infty} \mathrm{d} s \sigma(\gamma \gamma \rightarrow X) \frac{\mathrm{d} L}{\mathrm{~d} s}
$$

where $L=L_{\|}+L_{\perp}$.

When the non-electromagnetic interactions between the protons are neglected, $P(b)=1$, and the luminosities can be expressed through the integrated spectrum (2.3):

$$
\left.\frac{\mathrm{d} L_{\|}}{\mathrm{d} s}\right|_{P=1}=\left.\frac{\mathrm{d} L_{\perp}}{\mathrm{d} s}\right|_{P=1}=\left.\frac{1}{2} \frac{\mathrm{d} L}{\mathrm{~d} s}\right|_{P=1}=\frac{1}{8} \int_{-\infty}^{\infty} \mathrm{d} y n\left(\frac{\sqrt{s}}{2} \mathrm{e}^{y}\right) n\left(\frac{\sqrt{s}}{2} \mathrm{e}^{-y}\right) .
$$

Note that in this case the luminosities for different polarizations are equal, so one can use the simpler formula $(2.15)$ with $\sigma(\gamma \gamma \rightarrow X)=\frac{1}{2}\left(\sigma_{\|}(\gamma \gamma \rightarrow X)+\sigma_{\perp}(\gamma \gamma \rightarrow X)\right)$ to calculate the cross section. In other words, when the non-electromagnetic interactions between the colliding particles are neglected, so can be the polarization of photons. 
Ref. [25] suggests the following expression for $P(b)$ :

$$
P(b)=\left(1-\mathrm{e}^{-\frac{b^{2}}{2 B}}\right)^{2} .
$$

The parameter $B$ depends on the collision energy $E$. An empirical formula is provided in ref. [26]:

$$
B=B_{0}+2 B_{1} \ln \left(E / E_{0}\right)+4 B_{2} \ln ^{2}\left(E / E_{0}\right)
$$

with $B_{0}=12 \mathrm{GeV}^{-2}, B_{1}=-0.22 \pm 0.17 \mathrm{GeV}^{-2}, B_{2}=0.037 \pm 0.006 \mathrm{GeV}^{-2}, E_{0}=1 \mathrm{GeV}$. The validity of this formula was tested for $E=7$ and $8 \mathrm{TeV}[27,28]$ and was found to be in a good agreement with the experiment. For $E=13 \mathrm{TeV}$ we will use $B=21.1 \mathrm{GeV}^{-2}$.

Substituting (2.17) into (2.14) and integrating over the angles, we get

$$
\begin{aligned}
\frac{\mathrm{d} L_{\|}}{\mathrm{d} s}= & \frac{\pi^{2}}{2} \int_{\hat{r}}^{\infty} b_{1} \mathrm{~d} b_{1} \int_{\hat{r}}^{\infty} b_{2} \mathrm{~d} b_{2} \int_{-\infty}^{\infty} \mathrm{d} y n\left(b_{1}, \frac{\sqrt{s}}{2} \mathrm{e}^{y}\right) n\left(b_{2}, \frac{\sqrt{s}}{2} \mathrm{e}^{-y}\right) \\
& \times\left\{1-2 \mathrm{e}^{-\frac{b_{1}^{2}+b_{2}^{2}}{2 B}}\left[I_{0}\left(\frac{b_{1} b_{2}}{B}\right)+I_{2}\left(\frac{b_{1} b_{2}}{B}\right)\right]+\mathrm{e}^{-\frac{b_{1}^{2}+b_{2}^{2}}{B}}\left[I_{0}\left(\frac{2 b_{1} b_{2}}{B}\right)+I_{2}\left(\frac{2 b_{1} b_{2}}{B}\right)\right]\right\}, \\
\frac{\mathrm{d} L_{\perp}}{\mathrm{d} s}= & \frac{\pi^{2}}{2} \int_{\hat{r}}^{\infty} b_{1} \mathrm{~d} b_{1} \int_{\hat{r}}^{\infty} b_{2} \mathrm{~d} b_{2} \int_{-\infty}^{\infty} \mathrm{d} y n\left(b_{1}, \frac{\sqrt{s}}{2} \mathrm{e}^{y}\right) n\left(b_{2}, \frac{\sqrt{s}}{2} \mathrm{e}^{-y}\right) \\
& \times\left\{1-2 \mathrm{e}^{-\frac{b_{1}^{2}+b_{2}^{2}}{2 B}}\left[I_{0}\left(\frac{b_{1} b_{2}}{B}\right)-I_{2}\left(\frac{b_{1} b_{2}}{B}\right)\right]+\mathrm{e}^{-\frac{b_{1}^{2}+b_{2}^{2}}{B}}\left[I_{0}\left(\frac{2 b_{1} b_{2}}{B}\right)-I_{2}\left(\frac{2 b_{1} b_{2}}{B}\right)\right]\right\},
\end{aligned}
$$

where $I_{0}(z), I_{2}(z)$ are the modified Bessel functions of the first kind, and the equality

$$
\int_{0}^{\pi} \mathrm{e}^{z \cos \varphi} \cos (n \varphi) \mathrm{d} \varphi=\pi I_{n}(z)
$$

is used.

Survival factor describes the diminishing of the UPC cross section due to disintegration of the colliding particles. At the amplitude level, it is defined as the ratio of the cross sections with and without the survival effects included [29-34]:

$$
\left\langle S_{\gamma \gamma}\right\rangle=\frac{\int_{0}^{\infty} \mathrm{d} \omega_{1} \int_{0}^{\infty} \mathrm{d} \omega_{2} \int \mathrm{d}^{2} b_{1} \int \mathrm{d}^{2} b_{2} \sigma(\gamma \gamma \rightarrow X) n\left(b_{1}, \omega_{1}\right) n\left(b_{2}, \omega_{2}\right) P(b)}{\int_{0}^{\infty} \mathrm{d} \omega_{1} \int_{0}^{\infty} \mathrm{d} \omega_{2} \sigma(\gamma \gamma \rightarrow X) n\left(\omega_{1}\right) n\left(\omega_{2}\right)} .
$$

When the dependence of the $\gamma \gamma \rightarrow X$ cross section on the relative polarization of the photons is neglected, one gets the following expression for the survival factor: ${ }^{3}$

$$
S_{\gamma \gamma}=\frac{\mathrm{d} L / \mathrm{d} s \mathrm{~d} y}{\mathrm{~d} L /\left.\mathrm{d} s \mathrm{~d} y\right|_{P=1}}=\frac{\mathrm{d} L / \mathrm{d} \omega_{1} \mathrm{~d} \omega_{2}}{\mathrm{~d} L /\left.\mathrm{d} \omega_{1} \mathrm{~d} \omega_{2}\right|_{P=1}} .
$$

\footnotetext{
${ }^{3}$ In the literature, the survival factor is often denoted as $\left\langle S_{\gamma \gamma}\right\rangle^{2}, S_{\gamma \gamma}^{2}$.
} 
$S_{\gamma \gamma}$ depends on the invariant mass $\sqrt{s}$ and rapidity $y$ of the system produced or, equivalently, on the photons energies $\omega_{1}, \omega_{2}$. In the following we will use the definition of the survival factor with the luminosities integrated with respect to rapidity:

$$
S=\frac{\mathrm{d} L / \mathrm{d} s}{\mathrm{~d} L /\left.\mathrm{d} s\right|_{P=1}} .
$$

In the case of the production of a pair of charged fermions with the mass $m$, eqs. (2.22), (2.23) are valid for $s \gg m^{2}$, see eq. (3.1) in the next section.

\section{Production of heavy charged particles}

Results of calculations of photon-photon luminosities in proton-proton collisions with the energy $13 \mathrm{TeV}$ through eqs. (2.14), (2.16) are presented in figure 3 along with the survival factor (2.23). ${ }^{4}$ The difference between the luminosities of photons with parallel and perpendicular polarizations is below $10 \%$.

Production of a pair of charged fermions $\chi^{+} \chi^{-}$with the charge 1 and the mass $m_{\chi}$ is described by the Breit-Wheeler cross sections [35]:

$$
\begin{gathered}
\sigma_{\|}\left(\gamma \gamma \rightarrow \chi^{+} \chi^{-}\right)=\frac{4 \pi \alpha^{2}}{s}\left[\left(1+\frac{4 m_{\chi}^{2}}{s}-\frac{12 m_{\chi}^{4}}{s^{2}}\right) \ln \frac{1+\sqrt{1-4 m_{\chi}^{2} / s}}{1-\sqrt{1-4 m_{\chi}^{2} / s}}-\left(1+\frac{6 m_{\chi}^{2}}{s}\right) \sqrt{1-\frac{4 m_{\chi}^{2}}{s}}\right], \\
\sigma_{\perp}\left(\gamma \gamma \rightarrow \chi^{+} \chi^{-}\right)=\frac{4 \pi \alpha^{2}}{s}\left[\left(1+\frac{4 m_{\chi}^{2}}{s}-\frac{4 m_{\chi}^{4}}{s^{2}}\right) \ln \frac{1+\sqrt{1-4 m_{\chi}^{2} / s}}{1-\sqrt{1-4 m_{\chi}^{2} / s}}-\left(1+\frac{2 m_{\chi}^{2}}{s}\right) \sqrt{1-\frac{4 m_{\chi}^{2}}{s}}\right] .
\end{gathered}
$$

These formulae are substituted into eq. (2.13) for $X=\chi^{+} \chi^{-}$. The resulting cross section is shown in figure 4 .

In ref. [36] we propose a model-independent approach to the search of charged heavy long-lived particles produced in ultraperipheral collisions at the LHC. The corresponding cross section is presented in figure 2 of ref. [36]. ${ }^{5}$ From figure 4 we see that the cross section obtained in ref. [36] decreases due to proton disintegration by approximately $15 \%$ for $m_{\chi}=100 \mathrm{GeV}$. For heavier $\chi$ the decrease is larger according to the scaling of the survival factor by the variable $r_{p} \sqrt{s} / \gamma$ (heavier $\chi$ corresponds to larger $\sqrt{s}$ which has the same effect as making proton radius larger for fixed $m_{\chi}$ ).

Experimental data are often constrained by cuts on the phase space imposed to take into account detector geometry and to improve the signal to background ratio. Common requirements are for the particles produced to have transverse momentum $p_{T}>\hat{p}_{T}$ and pseudorapidity $|\eta|<\hat{\eta}$, where $\hat{p}_{T}, \hat{\eta}$ are the cut values, $\eta=-\ln \tan (\theta / 2), \theta$ is the angle between the momentum of the produced particle and the proton beam. The differential

\footnotetext{
${ }^{4}$ Ref. [24] argues that at large invariant masses $\mathrm{d} L_{\|} / \mathrm{d} s>\mathrm{d} L_{\perp} / \mathrm{d} s$. In proton-proton collisions with the energy $13 \mathrm{TeV}$ this transition occurs at $\sqrt{s} \sim 7 \mathrm{TeV}$

${ }^{5}$ In our calculations in ref. [36] we used $\Lambda^{2}=\Lambda_{\text {std }}^{2}=0.71 \mathrm{GeV}^{2}$ and neglected the magnetic component of the proton form factor.
} 


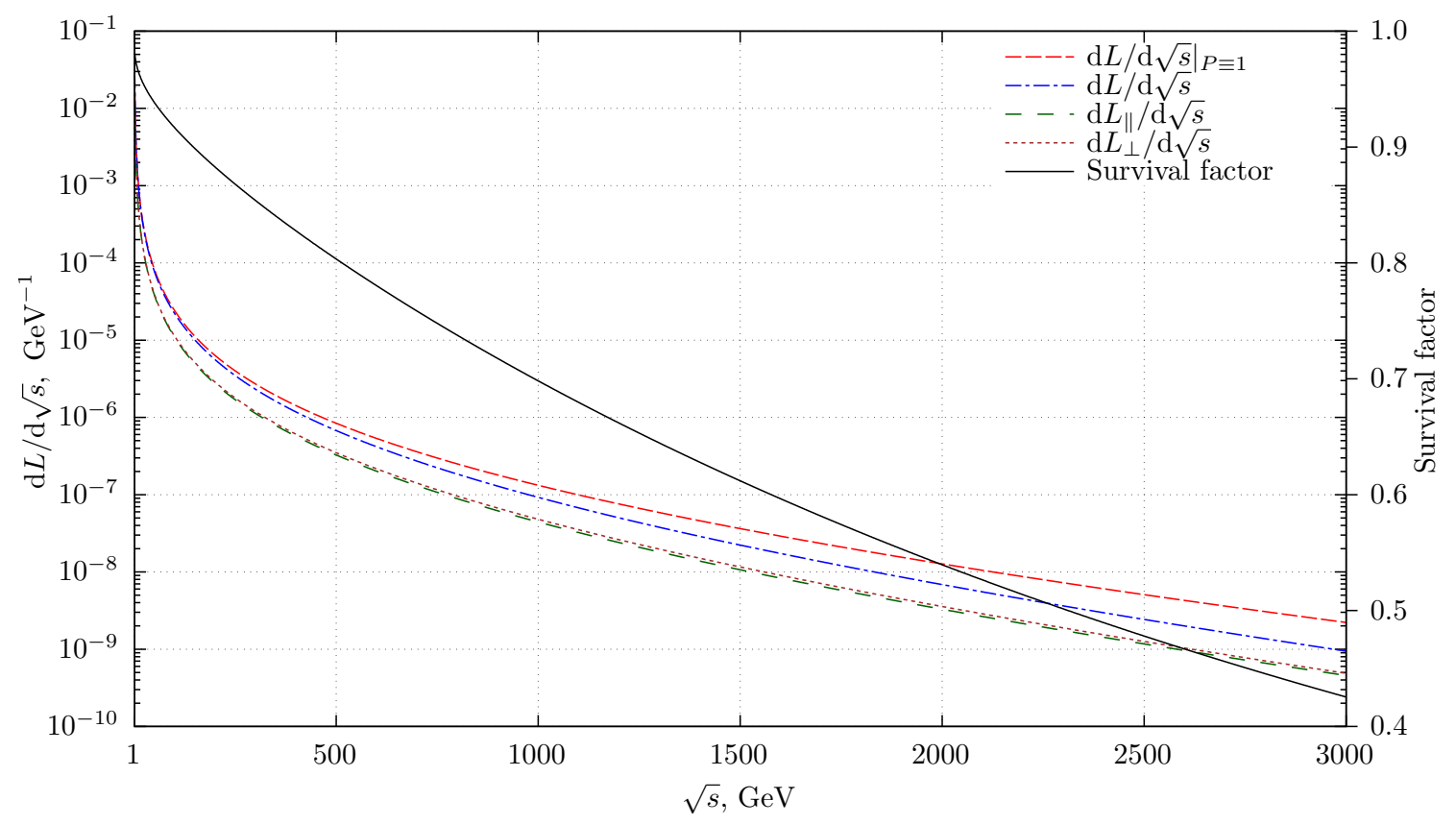

Figure 3. Survival factor $S$ (2.23) (right axis) and corresponding equivalent photon-photon luminosities (left axis) in $p p$ collisions with the energy $13 \mathrm{TeV}$. Here $\sqrt{s}$ is the invariant mass of the produced system.

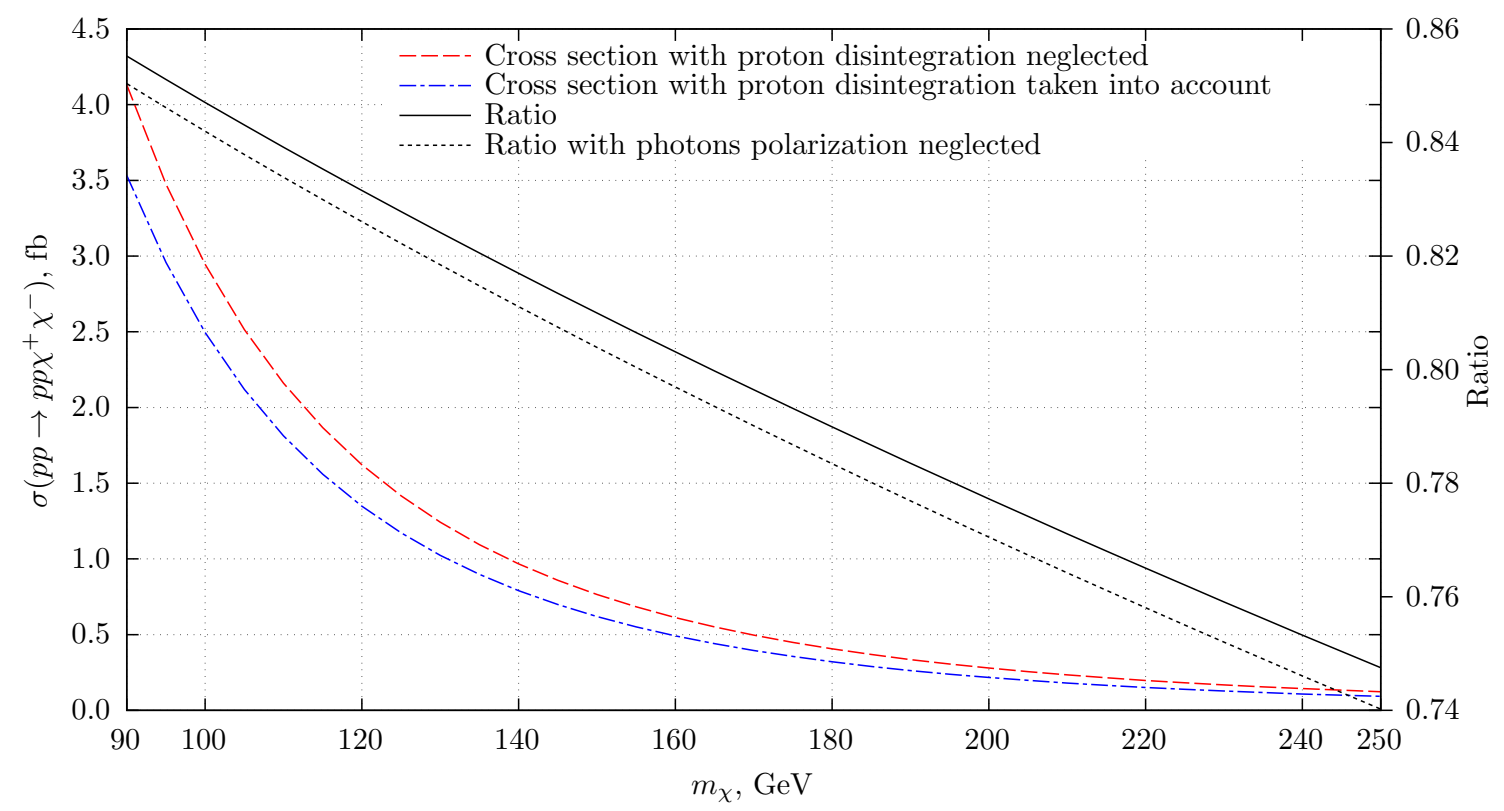

Figure 4. Cross sections (left axis) and their ratio (right axis) for the production of a pair of charged particles $\chi^{+} \chi^{-}$in $p p$ collisions with the energy $13 \mathrm{TeV}$ with respect to the particle mass. The black dotted line (right axis) is the ratio of the cross sections when the polarization of photons in UPC is neglected. The corresponding cross section is not shown because in this plot it is barely distinguishable from the dash-dotted line. 
fiducial cross section for the production of a $\chi^{+} \chi^{-}$pair is then

$$
\begin{aligned}
& \frac{\mathrm{d} \sigma_{\text {fid. }}\left(p p \rightarrow p p \chi^{+} \chi^{-}\right)}{\mathrm{d} s} \\
& =\int_{\max \left(\hat{p}_{T}, \frac{\sqrt{s}}{2 \cosh \hat{\eta}} \sqrt{1-\frac{4 m_{\chi}^{2}}{s}}\right)}^{\frac{\sqrt{s}}{2} \sqrt{1-\frac{4 m_{\chi}^{2}}{s}}} \mathrm{~d} p_{T}\left[\frac{\mathrm{d} \sigma_{\|}\left(\gamma \gamma \rightarrow \chi^{+} \chi^{-}\right)}{\mathrm{d} p_{T}} \frac{\mathrm{d} \hat{L}_{\|}}{\mathrm{d} s}+\frac{\mathrm{d} \sigma_{\perp}\left(\gamma \gamma \rightarrow \chi^{+} \chi^{-}\right)}{\mathrm{d} p_{T}} \frac{\mathrm{d} \hat{L}_{\perp}}{\mathrm{d} s}\right],
\end{aligned}
$$

where the differential with respect to $p_{T}$ cross sections are

$$
\begin{gathered}
\frac{\mathrm{d} \sigma_{\|}\left(\gamma \gamma \rightarrow \chi^{+} \chi^{-}\right)}{\mathrm{d} p_{T}}=\frac{8 \pi \alpha^{2} p_{T}}{s\left(p_{T}^{2}+m_{\chi}^{2}\right)} \cdot \frac{1-\frac{2\left(p_{T}^{4}+2 m_{\chi}^{4}\right)}{s\left(p_{T}^{2}+m_{\chi}^{2}\right)}}{\sqrt{1-\frac{4\left(p_{T}^{2}+m_{\chi}^{2}\right)}{s}}}, \\
\frac{\mathrm{d} \sigma_{\perp}\left(\gamma \gamma \rightarrow \chi^{+} \chi^{-}\right)}{\mathrm{d} p_{T}}=\frac{8 \pi \alpha^{2} p_{T}}{s\left(p_{T}^{2}+m_{\chi}^{2}\right)} \cdot \frac{1-\frac{2 p_{T}^{4}}{s\left(p_{T}^{2}+m_{\chi}^{2}\right)}}{\sqrt{1-\frac{4\left(p_{T}^{2}+m_{\chi}^{2}\right)}{s}}},
\end{gathered}
$$

and

$$
\begin{aligned}
\frac{\mathrm{d} \hat{L}_{\|}}{\mathrm{d} s} & =\frac{1}{4} \iint \mathrm{d}^{2} b_{1} \mathrm{~d}^{2} b_{2} P(b) \int_{-\hat{y}}^{\hat{y}} \mathrm{~d} y n\left(b_{1}, \frac{\sqrt{s}}{2} \mathrm{e}^{y}\right) n\left(b_{2}, \frac{\sqrt{s}}{2} \mathrm{e}^{-y}\right) \cos ^{2} \varphi, \\
\frac{\mathrm{d} \hat{L}_{\perp}}{\mathrm{d} s} & =\frac{1}{4} \iint \mathrm{d}^{2} b_{1} \mathrm{~d}^{2} b_{2} P(b) \int_{-\hat{y}}^{\hat{y}} \mathrm{~d} y n\left(b_{1}, \frac{\sqrt{s}}{2} \mathrm{e}^{y}\right) n\left(b_{2}, \frac{\sqrt{s}}{2} \mathrm{e}^{-y}\right) \sin ^{2} \varphi
\end{aligned}
$$

are the photon-photon luminosities (2.14) that take into account the cut on the pseudorapidity of the produced particles [36]:

$$
\hat{y}=\operatorname{arcsinh}\left[\frac{\sqrt{s} p_{T}}{2\left(p_{T}^{2}+m_{\chi}^{2}\right)}\left(\sinh \hat{\eta}-\sqrt{\cosh ^{2} \hat{\eta}+\frac{m_{\chi}^{2}}{p_{T}^{2}}} \cdot \sqrt{1-\frac{4\left(p_{T}^{2}+m_{\chi}^{2}\right)}{s}}\right)\right] .
$$

Another common constraint specific to ultraperipheral collisions is the requirement for the protons to hit the forward detectors. Forward detectors are special purpose detectors located at $\approx 200 \mathrm{~m}$ down the beam from both sides of the central detectors of the ATLAS and CMS collaborations [37, 38]. To hit a forward detector, a proton has to lose $1.5-15 \%$ of its energy which in the case of $13 \mathrm{TeV}$ collisions corresponds to photon energies from $\omega_{\min }=97.5 \mathrm{GeV}$ to $\omega_{\max }=975 \mathrm{GeV}$. To apply this constraint to the calculation of the 


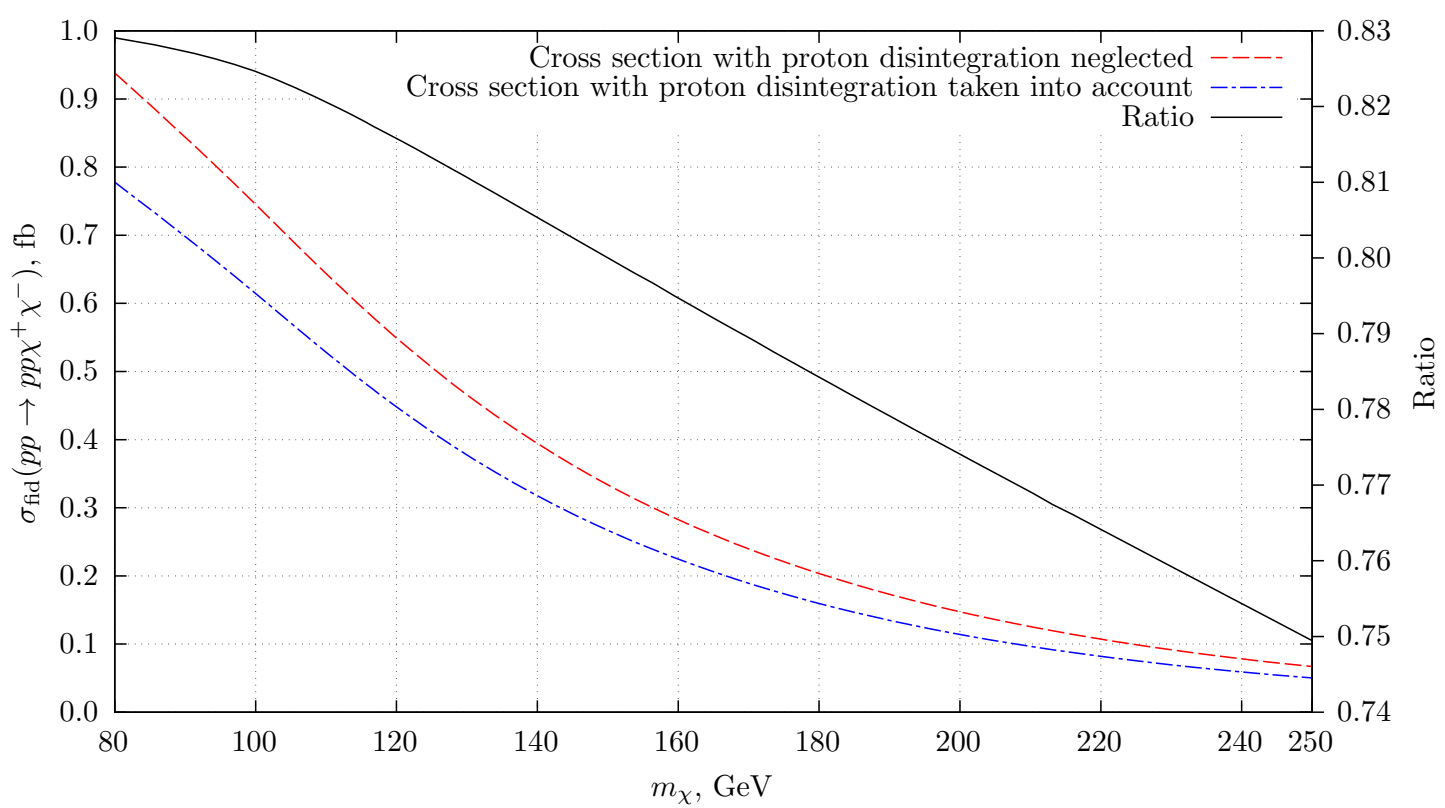

Figure 5. Cross sections (left axis) and their ratio (right axis) for the production of a pair of charged particles $\chi^{+} \chi^{-}$in $p p$ collisions with the energy $13 \mathrm{TeV}$ with respect to the particle mass. Both particles are required to have transverse momentum greater than $20 \mathrm{GeV}$ and pseudorapidity less than 2.5; both protons are required to hit the forward detectors.

differential fiducial cross section (3.2), integration limits in eq. (3.4) have to be modified:

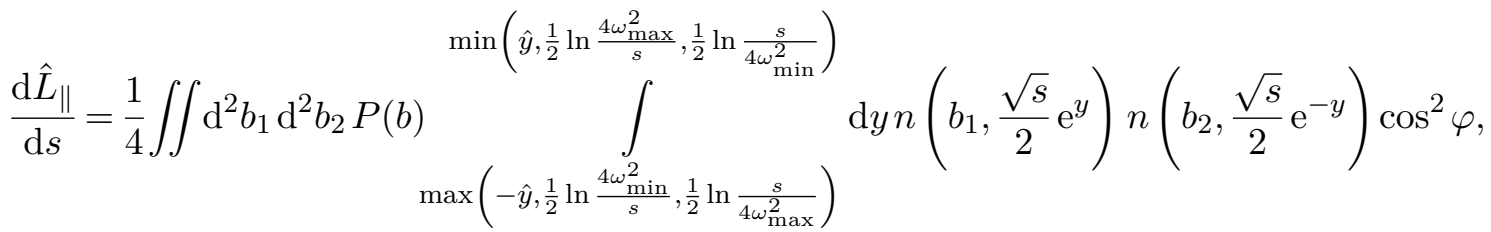

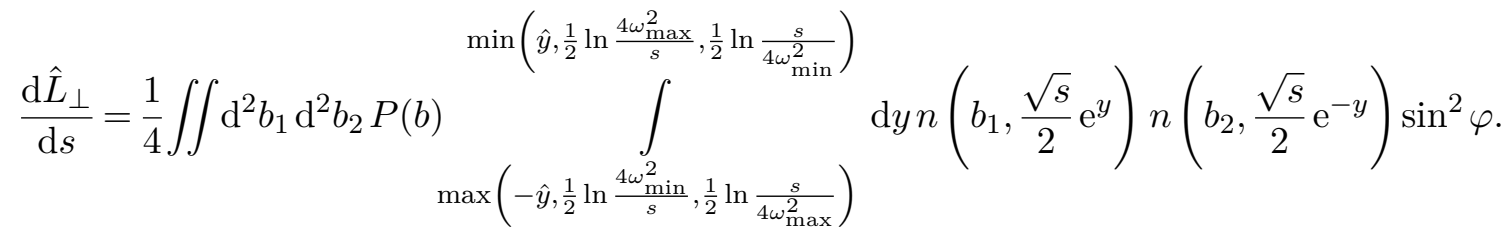

Integration of the differential fiducial cross section should be performed under the constraint $4 \omega_{\min }^{2} \leq s \leq 4 \omega_{\max }^{2}$. Cross section for the production of a pair of charged particles for $\hat{p}_{T}=20 \mathrm{GeV}, \hat{\eta}=2.5$ and under the requirement that both protons hit the forward detectors is presented in figure 5. For the muons, $\sigma_{\mathrm{fid}}\left(p p \rightarrow p p \mu^{+} \mu^{-}\right) \approx 1.4 \mathrm{fb}(1.7 \mathrm{fb})$ when the proton disintegration is taken into account (neglected) which gives the ratio 0.84. To be detected in a forward detector, a proton should emit a photon with the energy at least $97.5 \mathrm{GeV}$. That is why the ratio does not reach 1 with the diminishing of the produced particles mass. It can be clearly seen in figure 5 as solid black line approaches constant in the low mass region. 


\begin{tabular}{ccc}
\hline Muon pair invariant mass range & Muon transverse momentum & Muon pseudorapidity \\
\hline $12 \mathrm{GeV}<\sqrt{s}<30 \mathrm{GeV}$ & $p_{T}>6 \mathrm{GeV}$ & $|\eta|<2.4$ \\
$30 \mathrm{GeV}<\sqrt{s}<70 \mathrm{GeV}$ & $p_{T}>10 \mathrm{GeV}$ & \\
\hline
\end{tabular}

Table 1. Experimental cuts for the fiducial cross section of the $p p(\gamma \gamma) \rightarrow p p \mu^{+} \mu^{-}$reaction measured in ref. [39].

\section{Comparison to the experimental data}

In ref. [19], M.V. and E.Z. have provided an analytical description in terms of EPA of muon pair production in proton-proton UPC at the LHC neglecting the proton disintegration. They have compared their results to the experimental data obtained in ref. [39]. Let us demonstrate the effect of the survival factor on the description of the results of this experiment.

The experimental data are constrained with the cuts on the phase space $p_{T}>\hat{p}_{T}$, $|\eta|<\hat{\eta}$, where $p_{T}$ and $\eta$ are the transverse momentum and pseudorapidity of each muon. In the limit $p_{T} \gg m_{\mu}$, the differential cross sections $\mathrm{d} \sigma_{\|, \perp}\left(\gamma \gamma \rightarrow \mu^{+} \mu^{-}\right) / \mathrm{d} p_{T}$ for parallel and perpendicular photon linear polarizations (3.3) coincide, therefore the differential fiducial cross section $(3.2)$ is

$$
\frac{\mathrm{d} \sigma_{\text {fid }}\left(p p \rightarrow p p \mu^{+} \mu^{-}\right)}{\mathrm{d} s}=\int_{\max \left(\hat{p}_{T}, \frac{\sqrt{s}}{\cosh \hat{\eta}}\right)}^{\sqrt{s} / 2} \mathrm{~d} p_{T} \frac{\mathrm{d} \sigma\left(\gamma \gamma \rightarrow \mu^{+} \mu^{-}\right)}{\mathrm{d} p_{T}} \frac{\mathrm{d} \hat{L}}{\mathrm{~d} s},
$$

where $\hat{L}=\hat{L}_{\|}+\hat{L}_{\perp}$, and

$$
\frac{\mathrm{d} \sigma\left(\gamma \gamma \rightarrow \mu^{+} \mu^{-}\right)}{\mathrm{d} p_{T}}=\frac{8 \pi \alpha^{2}}{s p_{T}} \frac{1-2 p_{T}^{2} / s}{\sqrt{1-4 p_{T}^{2} / s}}
$$

Eq. (3.5) simplifies to

$$
\hat{y}=\hat{\eta}-\frac{1}{2} \ln \frac{1+\sqrt{1-4 p_{T}^{2} / s}}{1-\sqrt{1-4 p_{T}^{2} / s}} .
$$

The experimental cuts used in ref. [39] are described in table 1. The fiducial cross section calculated with the probability for the protons to survive during the collision (2.17) is plotted in figure $6 .^{6}$

The integrated fiducial cross section in the interval $12 \mathrm{GeV}<\sqrt{s}<70 \mathrm{GeV}$ is

- experimental value: $3.12 \pm 0.07$ (stat.) \pm 0.10 (syst.) pb [39];

- with proton disintegration neglected: $3.39 \mathrm{pb}$.

\footnotetext{
${ }^{6}$ Note that in our calculations in ref. [19] we used $\Lambda^{2}=\Lambda_{\mathrm{std}}^{2}=0.71 \mathrm{GeV}^{2}$ and neglected the magnetic component of the proton form factor. All three corrections: the magnetic form factor contribution, the change of $\Lambda^{2}$ from $\Lambda_{\text {std }}^{2}$ to $\Lambda_{\text {CODATA }}^{2}$, and the survival factor contribution have approximately the same magnitude in this experiment, with the former being positive and the other two negative.
} 


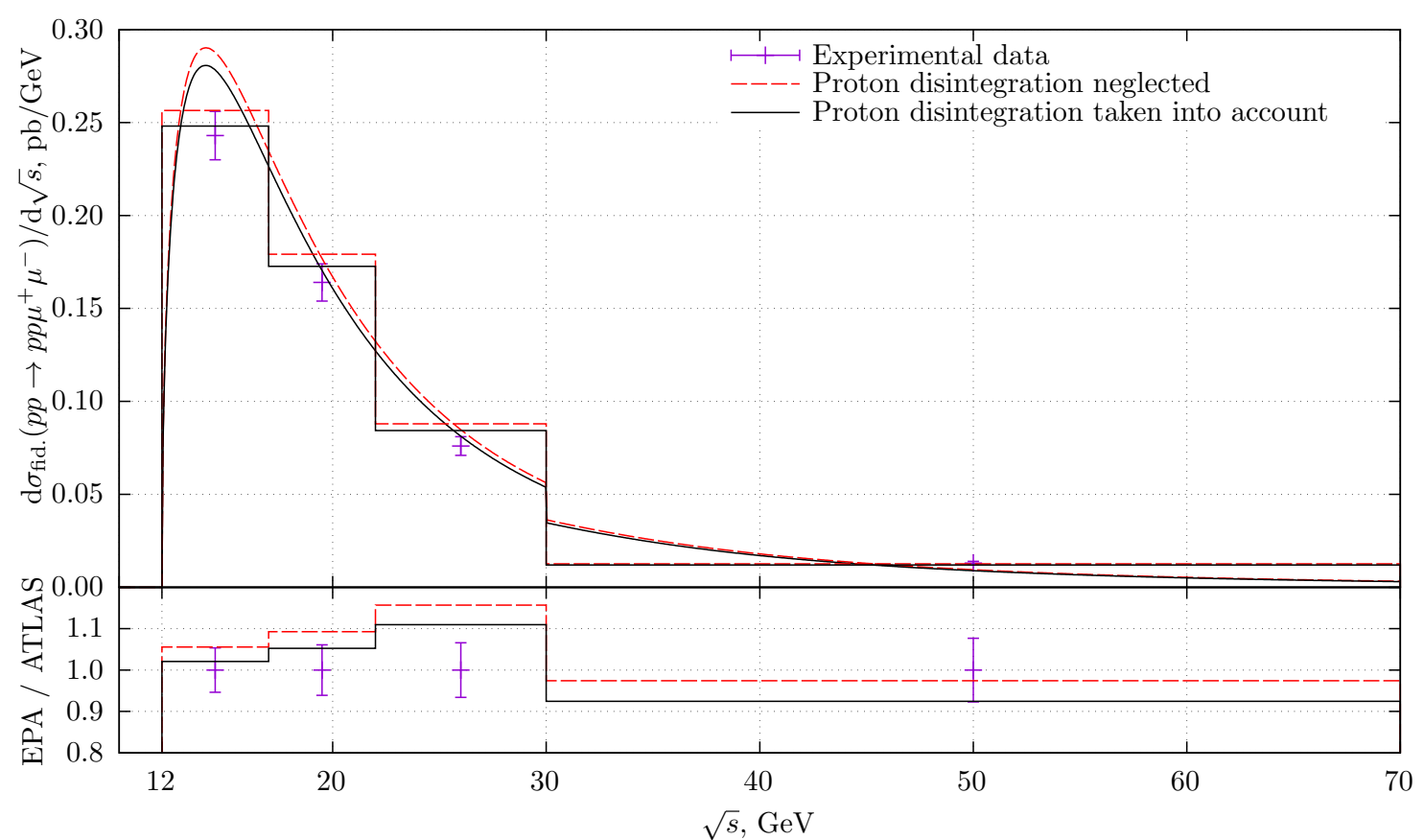

Figure 6. Upper plot: fiducial cross section of the $p p \rightarrow p p \mu^{+} \mu^{-}$reaction with the collision energy $13 \mathrm{TeV}$ and the cuts described in table 1 . The points are the experimental data from ref. [39]. The red dashed line is the differential cross section with the proton disintegration probability neglected. The black solid line is the differential cross section with the survival factor taken into account. The histograms are the cross sections integrated over the experimental bins. Lower plot: ratio of the calculated cross section to the experimental data.

- with proton disintegration taken into account: $3.26 \mathrm{pb}^{7}$

Ref. [39] also presents EPA predictions made with Monte Carlo simulations:

- Herwig [40, 41]: $3.56 \pm 0.05 \mathrm{pb}$;

- HeRwiG with corrections from ref. [42]: $3.06 \pm 0.05$ pb;

- SuperChic2 [32]: $3.45 \pm 0.05 \mathrm{pb}$.

Our result for the survival factor for the integrated cross section, $\left\langle S_{\gamma \gamma}\right\rangle=3.26 \mathrm{pb} / 3.39 \mathrm{pb}=$ 0.96 is the same as in table 2 of ref. [43]: $3.43 \mathrm{pb} / 3.58 \mathrm{pb}=0.96$.

\section{Conclusion}

The luminosity of photons appearing in ultraperipheral collisions of protons with the energy $13 \mathrm{TeV}$ is plotted in figure 3 along with the survival factor. There is little point in going beyond the presented range of invariant masses, because a proton will not survive emitting

\footnotetext{
${ }^{7}$ Calculation with the proton form factor provided numerically by the A1 collaboration [23] (see supplementary materials) may result in the cross section $\approx 2 \%$ greater, see footnote 2 on page 3 .
} 


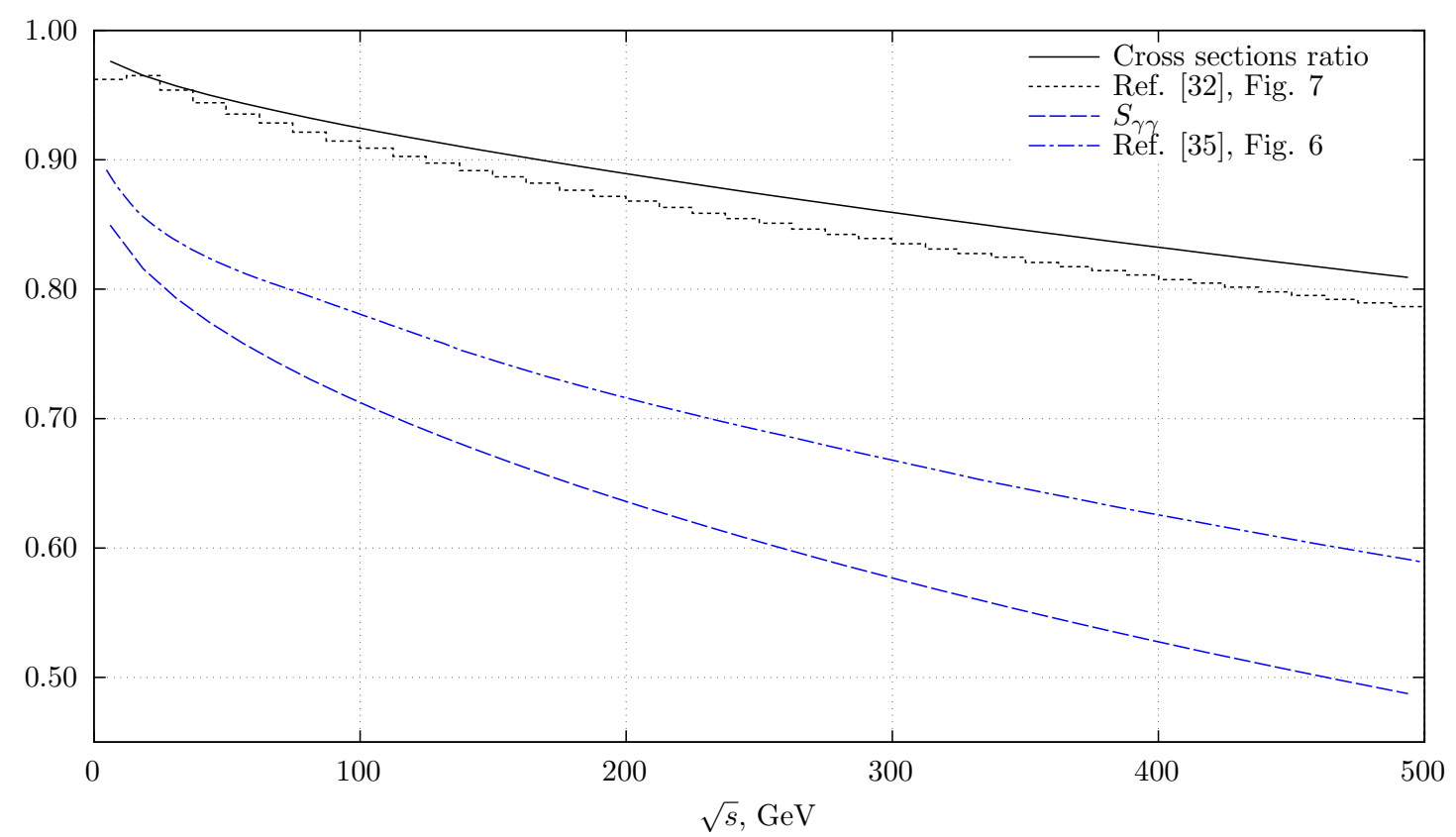

Figure 7. Comparison to the results obtained in refs. [32, 42]. The upper solid line is the ratio $\frac{\mathrm{d} \sigma_{\text {fid. }}\left(p p \rightarrow p p \mu^{+} \mu^{-}\right) / \mathrm{d} \sqrt{s}}{\mathrm{~d} \sigma_{\text {fid. }}\left(p p \rightarrow p p \mu^{+} \mu^{-}\right) /\left.\mathrm{d} \sqrt{s}\right|_{P=1}}$, where $\sigma_{\text {fid. }}\left(p p \rightarrow p p \mu^{+} \mu^{-}\right)$is the fiducial cross section (4.1) with the parameters $\hat{p}_{T}=2.5 \mathrm{GeV}, \hat{\eta}=2.5$ for the $p p$ collision energy $14 \mathrm{TeV} ; \sqrt{s}$ is the invariant mass of the muon pair. It is compared to the histogram representing the same value plotted in ref. [32]. The lower dashed line is the survival factor $S_{\gamma \gamma}(2.22)$ for the collision energy $13 \mathrm{TeV}$, rapidity $y=0$ and $\hat{r}=r_{p}$. It is compared to the dash-dotted line showing the survival factor from ref. [42]. ${ }^{8}$ The lines were extracted from the papers with the help of ref. [44].

a photon with the energy above $\sim 1.4 \mathrm{TeV}[19]$. Although the photons in UPC are polarized, the difference in the luminosities for different polarization arrangements is at the level of a few percent, resulting in negligible difference in the cross section for the production of a pair of charged fermions, see figure 4 .

Taking into account the survival factor improves the agreement between the theoretical description of the ATLAS experiment and the experimental data, see figure 6 .

In figure 7, we compare our calculations with the results obtained in refs. [32, 42]. The reduction of the cross section according to ref. [42] is much larger due to the restriction $b_{i}>r_{p}$ (or $\left.\hat{r}=r_{p}\right)$ imposed in ref. [42]. We did not impose such a restriction since diminishing of the photon flux at $b_{i}<r_{p}$ is taken into account by the elastic form factor, and the produced particles do not interact strongly with the protons. The numerical difference with the results obtained in [32] is marginal.

We are grateful to V. A. Khoze for the comments on photons polarizations in UPC collisions and the references to previous work. The authors were supported by the Russian Science Foundation grant No 19-12-00123.

\footnotetext{
${ }^{8}$ Note that the constraint $\hat{r}=r_{p}$ applied in ref. [42] is incorrect in the case of muon pair production, see the discussion after eq. (2.12) and ref. [43].
} 
Open Access. This article is distributed under the terms of the Creative Commons Attribution License (CC-BY 4.0), which permits any use, distribution and reproduction in any medium, provided the original author(s) and source are credited.

\section{References}

[1] C.A. Bertulani, G. Baur, Electromagnetic processes in relativistic heavy ion collisions, Phys. Rept. 163 (1988) 299.

[2] G. Baur, K. Hencken, D. Trautmann, S. Sadovsky and Y. Kharlov, Coherent $\gamma \gamma$ and $\gamma A$ interactions in very peripheral collisions at relativistic ion colliders, Phys. Rept. 364 (2002) 359 [hep-ph/0112211] [INSPIRE].

[3] G. Baur, Physics opportunities in ultraperipheral heavy ion collisions at LHC, in Workshop on Electromagnetic Probes of Fundamental Physics, (2001), pp. 183-191 [hep-ph/0112239] [INSPIRE].

[4] G. Baur et al., Hot topics in ultraperipheral ion collisions, in Workshop on Electromagnetic Probes of Fundamental Physics, (2002), pp. 235-241, DOI [hep-ex/0201034] [INSPIRE].

[5] L. Frankfurt, M. Strikman and M. Zhalov, Coherent photoproduction from nuclei, Acta Phys. Polon. B 34 (2003) 3215 [hep-ph/0304301] [INSPIRE].

[6] C.A. Bertulani, S.R. Klein and J. Nystrand, Physics of ultra-peripheral nuclear collisions, Ann. Rev. Nucl. Part. Sci. 55 (2005) 271 [nucl-ex/0502005] [INSPIRE].

[7] J. Nystrand, Ultra-peripheral collisions of heavy ions at RHIC and the LHC, Nucl. Phys. A 787 (2007) 29 [hep-ph/0611042] [INSPIRE].

[8] A.J. Baltz et al., The Physics of Ultraperipheral Collisions at the LHC, Phys. Rept. 458 (2008) 1 [arXiv:0706.3356] [INSPIRE].

[9] G. Baur, Coherent photon-photon interactions in very peripheral relativistic heavy ion collisions, Eur. Phys. J. D 55 (2009) 265 [arXiv:0810.1400] [InSPIRE].

[10] M. Kłusek-Gawenda and A. Szczurek, Exclusive production of large invariant mass pion pairs in ultraperipheral ultrarelativistic heavy ion collisions, Phys. Lett. B 700 (2011) 322 [arXiv: 1104.0571] [INSPIRE].

[11] A. Szczurek, Peripheral, ultrarelativistic production of particles in heavy ion collisions, Acta Phys. Polon. B 45 (2014) 1597 [arXiv: 1404.0896] [inSPIRE].

[12] M. Kłusek-Gawenda, P. Lebiedowicz and A. Szczurek, Light-by-light scattering in ultraperipheral $\mathrm{Pb}-\mathrm{Pb}$ collisions at energies available at the CERN Large Hadron Collider, Phys. Rev. C 93 (2016) 044907 [arXiv: 1601.07001] [INSPIRE].

[13] M. Kłusek-Gawenda and A. Szczurek, Double scattering production of two positron-electron pairs in ultraperipheral heavy-ion collisions, Phys. Lett. B 763 (2016) 416 [arXiv: 1607.05095] [INSPIRE].

[14] M.B. Gay Ducati, F. Kopp, M.V.T. Machado and S. Martins, Photoproduction of Upsilon states in ultraperipheral collisions at the CERN Large Hadron Collider within the color dipole approach, Phys. Rev. D 94 (2016) 094023 [arXiv: 1610.06647] [INSPIRE].

[15] M. Kłusek-Gawenda, P. Lebiedowicz, O. Nachtmann and A. Szczurek, From the $\gamma \gamma \rightarrow p \bar{p}$ reaction to the production of $p \bar{p}$ pairs in ultraperipheral ultrarelativistic heavy-ion collisions at the LHC, Phys. Rev. D 96 (2017) 094029 [arXiv:1708.09836] [INSPIRE]. 
[16] R.N. Cahn and J.D. Jackson, Realistic equivalent photon yields in heavy ion collisions, Phys. Rev. D 42 (1990) 3690 [INSPIRE].

[17] M. Vidović, M. Greiner, C. Best and G. Soff, Impact parameter dependence of the electromagnetic particle production in ultrarelativistic heavy ion collisions, Phys. Rev. C $\mathbf{4 7}$ (1993) 2308 [INSPIRE].

[18] M. Vidović, M. Greiner and G. Soff, Electromagnetic dissociation of Pb nuclei in peripheral ultrarelativistic heavy ion collisions, Phys. Rev. C 48 (1993) 2011 [InSPIRE].

[19] M.I. Vysotsky and E.V Zhemchugov, Equivalent photons in proton-proton and ion-ion collisions at the Large Hadron Collider, Phys. Usp. 62 (2019) 910 [arXiv:1806.07238] [INSPIRE].

[20] F.J. Ernst, R.G. Sachs and K.C. Wali, Electromagnetic form factors of the nucleon, Phys. Rev. 119 (1960) 1105 [INSPIRE].

[21] S. Pacetti, R.B. Ferroli, E. Tomasi-Gustafsson, Proton electromagnetic form factors: basic notions, present achievements and future perspectives, Phys. Rept. 550 (2015) 1.

[22] P.J. Mohr, D.B. Newell and B.N. Taylor, CODATA Recommended Values of the Fundamental Physical Constants: 2014, Rev. Mod. Phys. 88 (2016) 035009 [arXiv:1507.07956] [InSPIRE].

[23] A1 collaboration, Electric and magnetic form factors of the proton, Phys. Rev. C 90 (2014) 015206 [arXiv: 1307.6227] [INSPIRE].

[24] G. Baur and L.G. Ferreira Filho, Coherent particle production at relativistic heavy-ion colliders including strong absorption effects, Nucl. Phys. A $\mathbf{5 1 8}$ (1990) 786 [INSPIRE].

[25] L. Frankfurt, C.E. Hyde, M. Strikman and C. Weiss, Generalized parton distributions and rapidity gap survival in exclusive diffractive pp scattering, Phys. Rev. D 75 (2007) 054009 [hep-ph/0608271] [INSPIRE].

[26] V.A. Schegelsky and M.G. Ryskin, The diffraction cone shrinkage speed up with the collision energy, Phys. Rev. D 85 (2012) 094024 [arXiv:1112.3243] [INSPIRE].

[27] ATLAS collaboration, Measurement of the total cross section from elastic scattering in pp collisions at $\sqrt{s}=7 \mathrm{TeV}$ with the ATLAS detector, Nucl. Phys. B $\mathbf{8 8 9}$ (2014) 486 [arXiv: 1408.5778] [INSPIRE].

[28] ATLAS collaboration, Measurement of the total cross section from elastic scattering in pp collisions at $\sqrt{s}=8 \mathrm{TeV}$ with the ATLAS detector, Phys. Lett. B 761 (2016) 158 [arXiv: 1607.06605] [INSPIRE].

[29] V.A. Khoze, A.D. Martin, R. Orava and M.G. Ryskin, Luminosity monitors at the LHC, Eur. Phys. J. C 19 (2001) 313 [hep-ph/0010163] [INSPIRE].

[30] V.A. Khoze, A.D. Martin and M.G. Ryskin, Photon exchange processes at hadron colliders as a probe of the dynamics of diffraction, Eur. Phys. J. C 24 (2002) 459 [hep-ph/0201301] [INSPIRE].

[31] L.A. Harland-Lang, V.A. Khoze, M.G. Ryskin and W.J. Stirling, Central exclusive production within the Durham model: a review, Int. J. Mod. Phys. A 29 (2014) 1430031 [arXiv: 1405.0018] [INSPIRE].

[32] L.A. Harland-Lang, V.A. Khoze and M.G. Ryskin, Exclusive physics at the LHC with SuperChic 2, Eur. Phys. J. C 76 (2016) 9 [arXiv: 1508. 02718] [inSPIRE]. 
[33] V.A. Khoze, A.D. Martin and M.G. Ryskin, Multiple interactions and rapidity gap survival, J. Phys. G 45 (2018) 053002 [arXiv:1710.11505] [inSPIRE].

[34] L.A. Harland-Lang, M. Tasevsky, V.A. Khoze and M.G. Ryskin, A new approach to modelling elastic and inelastic photon-initiated production at the LHC: SuperChic 4, Eur. Phys. J. C 80 (2020) 925 [arXiv:2007.12704] [INSPIRE].

[35] G. Breit and J.A. Wheeler, Collision of two light quanta, Phys. Rev. 46 (1934) 1087 [INSPIRE].

[36] S.I. Godunov, V.A. Novikov, A.N. Rozanov, M.I. Vysotsky and E.V. Zhemchugov, Quasistable charginos in ultraperipheral proton-proton collisions at the LHC, JHEP 01 (2020) 143 [arXiv: 1906. 08568] [INSPIRE].

[37] L. Adamczyk et al., Technical Design Report for the ATLAS Forward Proton Detector, Tech. Rep. CERN-LHCC-2015-009, ATLAS-TDR-024 (2015).

[38] M. Albrow et al., CMS-TOTEM Precision Proton Spectrometer, Tech. Rep. CERN-LHCC-2014-021, TOTEM-TDR-003, CMS-TDR-13 (2014).

[39] ATLAS collaboration, Measurement of the exclusive $\gamma \gamma \rightarrow \mu^{+} \mu^{-}$process in proton-proton collisions at $\sqrt{s}=13 \mathrm{TeV}$ with the ATLAS detector, Phys. Lett. B 777 (2018) 303 [arXiv: 1708.04053] [INSPIRE].

[40] M. Bähr et al., HERWIG++ Physics and Manual, Eur. Phys. J. C 58 (2008) 639 [arXiv: 0803.0883] [INSPIRE].

[41] J. Bellm et al., HERWIG 7.0/HERWIG++ 3.0 release note, Eur. Phys. J. C 76 (2016) 196 [arXiv: 1512.01178] [INSPIRE].

[42] M. Dyndal and L. Schoeffel, The role of finite-size effects on the spectrum of equivalent photons in proton-proton collisions at the LHC, Phys. Lett. B 741 (2015) 66 [arXiv: 1410.2983] [INSPIRE].

[43] L.A. Harland-Lang, V.A. Khoze and M.G. Ryskin, Elastic photon-initiated production at the LHC: the role of hadron-hadron interactions, SciPost Phys. 11 (2021) 064 [arXiv:2104.13392] [INSPIRE].

[44] A. Rohatgi. WebPlotDigitizer - Web Based Plot Digitizer, https://automeris.io/WebPlotDigitizer. 